\title{
Empirische Beobachtungen zur Akzeptanz des Pilotprojektes „Autonom fahrender Kleinbus" unter den Bürger*innen von Bad Birnbach
}

\author{
Jürgen Rauh, Alexandra Appel und Maximilian GraßI
}

In dem Beitrag werden auf der Grundlage einer Haushaltsbefragung in Bad Birnbach Aussagen zur Akzeptanz, Aufgeschlossenheit und Bewertung gegenüber dem Pilotprojekt getroffen. Die Wahrnehmung und Bewertung von Eigenschaften des automatisiert fahrenden Kleinbusses wie auch Präferenzen, Werte sowie Einstellungen von Anwohnern zu autonom fahrenden Fahrzeugen sind dabei von Interesse. Als Analyseschema wird eine Einteilung nach den Akzeptanzdimensionen der Einstellungs-, Handlungs- und Nutzungsebene verwendet. Das Pilotprojekt wird von der Mehrheit der Befragten begrüßt und häufig auch schon aktiv getestet. Hinsichtlich einiger objekt- wie auch subjektbezogener Eigenschaften gibt es z. T. signifikante Unterschiede zwischen Personen, die schon mit dem automatisierten Bus mitgefahren sind, und solchen, die ihn noch nicht getestet haben. In Bezug auf eine zukünftige mögliche Nutzung können projektbezogen drei verschiedene Gruppen von potenziellen Nutzern differenziert werden.

\subsection{Hintergrund und Zielsetzung}

Die Verkehrs- und Mobilitätssituation in ländlich-peripheren Räumen stellt ein großes und viel diskutiertes gesellschaftliches Problem dar. Neue Angebotsformen und Innovationen im Verkehrsbereich erfahren vorwiegend in urbanen Räumen ihre ersten Anwendungen, während in ländlich-peripheren Räumen die Nachfragedichten zu gering sind, um bei betriebswirtschaftlichen Kosten-Nutzen-Kalkulationen auch nur annähernd Wettbewerbsfä-

J. Rauh $(\bowtie) \cdot$ A. Appel $\cdot$ M. Graß1

Julius-Maximilians-Universität Würzburg, Würzburg, Deutschland

E-Mail: juergen.rauh@uni-wuerzburg.de; alexandra.appel@uni-wuerzburg.de; maximilian.

grassl@stud-mail.uni-wuerzburg.de 
higkeit zu erreichen. Eine Einführung neuer Technologien und Systeme in ländlichen Räumen erfolgt daher gar nicht oder mit großer zeitlicher Verzögerung.

Autonom fahrende Kleinbusse ${ }^{1}$ böten jedoch aufgrund möglicher Einsparungen von Personal- und Energiekosten zumindest das Potenzial, um zu einer Problemlösung in der Bedienung mit öffentlichem Personennahverkehr (ÖPNV) des ländlichen Raumes beizutragen. Dieser erwünschte Effekt kann sich dauerhaft aber nur einstellen, wenn auch eine entsprechende Akzeptanz sowie Nachfrage nach autonom fahrenden Bussen durch die in ländlichen Räumen wohnende Bevölkerung besteht.

Das Pilotprojekt „Autonom fahrender Kleinbus“ bietet der Begleitforschung die Möglichkeit, die bisherige Bewertung des Projektes sowie das Testen und Ausprobieren durch die Anwohner zu analysieren. Es lassen sich zudem entlang verschiedener Dimensionen der Akzeptanz Hintergründe für eine Annahme oder Ablehnung bezogen auf das Pilotprojekt untersuchen und Erkenntnisse hinsichtlich einer Akzeptanz und potenziellen Nutzung zukünftiger autonomer Bussysteme in ländlichen Räumen gewinnen. Dabei steht weniger die Betrachtung (alltäglicher) Mobilitätsroutinen im Fokus des Interesses, sondern vielmehr Kriterien der objekt- und subjektbezogenen Einstellungen und Handlungen. Die Wahrnehmung und Bewertung von Eigenschaften des autonom fahrenden Kleinbusses wie auch Präferenzen, Werte sowie Einstellungen von Bürgern zu automatisierten Fahrzeugen sowie zum Pilotprojekt selbst sind dabei von Interesse. Letztendlich können so begünstigende wie hemmende Faktoren für potenzielle zukünftige Nutzungen, die über das Pilotprojekt hinausgehen, identifiziert werden.

Einzuordnen ist der Beitrag damit in den weiten Bereich der Technikakzeptanzforschung. Zur Akzeptanz neuer (Verkehrs-)Technologien gibt es aus unterschiedlichen sozial-, wirtschafts- und ingenieurwissenschaftlichen Disziplinen entsprechende Zugänge und Ansätze. Eine gängige begriffliche Differenzierung ist die Unterscheidung nach Akzeptanzobjekt, Akzeptanzsubjekt und Akzeptanzkontext (Lucke 1995, S. 88 f.; Schäfer und Keppler 2013, S. 16; Fraedrich und Lenz 2015, S. 642 f.). Im Folgenden sind als Akzeptanzobjekt das automatisiert fahrende Kleinbussystem in Bad Birnbach, als -subjekt die Bürger von Bad Birnbach ${ }^{2}$ und als -kontext die Rahmen- und Ausgangsbedingungen des Projektes zu verstehen.

Eine weitere Art, um Akzeptanz zu differenzieren, ist die Einteilung nach Dimensionen (z. B. Hüsing et al. 2002, S. 23; Schäfer und Keppler 2013; Fraedrich und Lenz 2015, S. 643 f.). Sie bietet die Möglichkeit, objekt- oder kontextbezogene Faktoren, welche Einfluss auf die Akzeptanz nehmen, zu strukturieren und z. B. in Input-, Input-Output-, Rück-

\footnotetext{
${ }^{1}$ Im Folgenden wird an mehreren Stellen der Terminus ,,autonom fahrender Kleinbus“ verwendet, obwohl es sich beim Pilotprojekt in technisch-organisatorischer Hinsicht um ein automatisiertes Kleinbussystem handelt. In der öffentlichen Kommunikation ist jedoch vorwiegend vom autonom fahrenden Kleinbus bzw. Shuttlebus die Rede, sodass auch der Verständlichkeit halber in der Befragung und im Fragebogen dieser Terminus verwendet wurde. Als Kürzel für den automatisierten Kleinbus wird im Analyseteil auch A.B. verwendet.

${ }^{2}$ Touristen und Kurgäste als weitere potenzielle größere Nutzergruppe werden im Folgenden nicht behandelt.
} 
kopplungs- und Phasenmodelle (Schäfer und Keppler 2013, S. 25 ff.) darzustellen. Häufig erfolgt eine Differenzierung nach Einstellungs-, Handlungs- sowie Wert- und Zieldimension (z. B. Kollmann 1998, S. 108; Hüsing et al. 2002, S. 23; Fraedrich und Lenz 2015, S. 643 f.). Schweizer-Ries und Rau (2010, S. 12) differenzieren zwischen einer Bewertungsebene und einer Handlungsebene, die sie auch als aktive Akzeptanz bezeichnen. Die Akzeptanz eines Akzeptanzobjektes ,stellt das positive, zeitlich relativ konstante Ergebnis eines an bestimmte Rahmenbedingungen (Kontextfaktoren) geknüpften Bewertungsprozesses durch ein Akzeptanzsubjekt [...] dar (=Bewertungsebene). Diese positive Bewertung kann zudem mit einer diesem Bewertungsurteil und dem wahrgenommenen Handlungsrahmen (-möglichkeiten) entsprechenden Handlungsabsicht bis hin zu konkreten unterstützenden Handlungen einhergehen (=Handlungsebene)“ (ebd.).

Als Analyseschema für den empirischen Teil soll die Akzeptanz des automatisiert fahrenden Kleinbusses durch die Bürger nach den Dimensionen der Einstellungs-, Handlungs- und Nutzungsebene geschehen (Kollmann 1998; Wisser 2018, S. 48 ff.). Dieser Einteilung liegt das Phasenmodell von Kollmann (1998, S. 113) zugrunde. Dieses lässt sich weiter differenzieren und in Zusammenhang mit der ,Entfaltung erwünschter Wirkungen neuer Technologien und Innovationen" (Hüsing et al. 2002, S. 1) bringen (Abb. 9.1). Auf eine technische Neuerung in seiner frühen Entwicklungsphase, wie es beim Pilotprojekt der Fall ist, folgt auf Subjektseite eine kognitive Reaktion. Dieser Wahrnehmung schließt sich eine Bewertung an: Kollmann (2016, S. 411) sieht auf der Einstellungsebene eine Verknüpfung von Wert- und Zielvorstellungen mit der rationalen Handlungsbereitschaft. Die Einstellung gegenüber der neuen Technologie ist ein zentrales Kriterium der Akzeptanz, nicht nur, weil sie in jeder Akzeptanzdefinition Berücksichtigung findet (Schäfer und Keppler 2013, S. 11), sondern weil sie wechselseitig in Bezug mit der Handlungsdimension steht, was Schweizer-Ries und Rau (2010, S. 11) auch in einem zweidimensionalen Modell darstellen. Dieser Bewertungsprozess ist an bestimmte Rah-

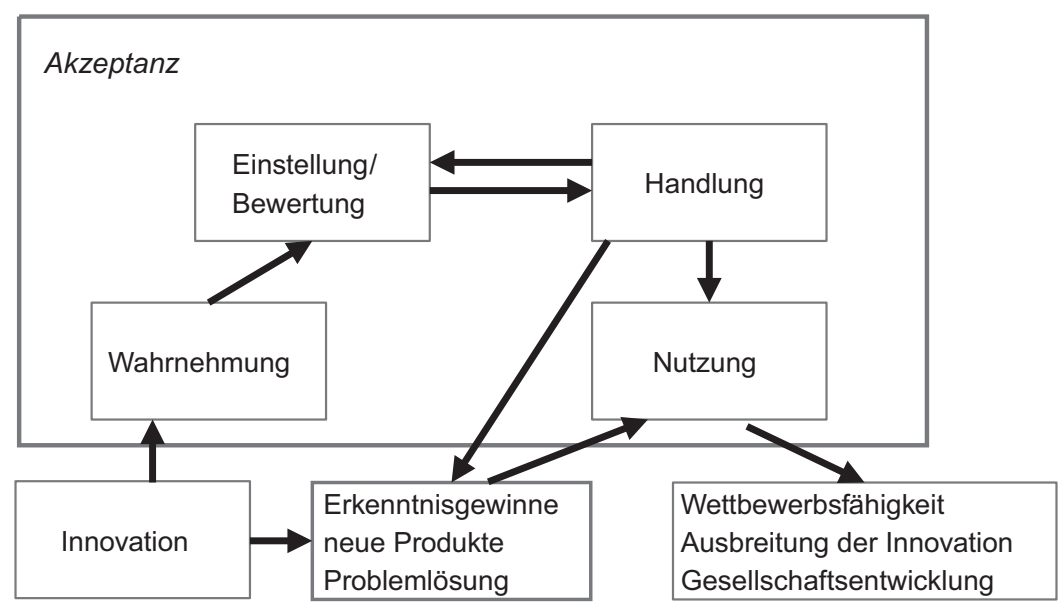

Abb. 9.1 Akzeptanz und Verbreitung neuer Technologien. (Quelle: eigene Darstellung, verändert nach Hüsing et al. [2002, S. 1]) 
menbedingungen geknüpft (Schweizer-Ries und Rau 2010, S. 12) und demnach auch in Kontexten zu analysieren (Fraedrich und Lenz 2015, S. 644). Haltungen, Wertungen und Einschätzungen der Bürger zum automatisiert fahrenden Kleinbus in Bad Birnbach sowie zu Mobilitätsfragen genereller Art sollen vor allem daraufhin betrachtet werden, wie sie im Zusammenhang mit Befürwortung oder Ablehnung des Pilotprojektes sowie dem aktiven „Ausprobieren“ des Kleinbusses stehen (Handlungsebene). Erfahrungen aus dem aktiven Testen des Pilotprojektes können nicht nur Effekte auf die Ausgestaltung und Verbesserung des Produktes „Autonomer Kleinbus“, sondern auch auf spätere Nutzungen im Regulärbetrieb haben. Entsprechend ausgereifte Produkte können wettbewerbsfähig sein und räumlich diffundieren.

\subsection{Methodisches Vorgehen und Beschreibung der Stichprobe}

Die beschriebenen Dimensionen sowie Faktoren von Akzeptanz und Nutzung wurden in einem standardisierten Fragebogen abgebildet, der sich an die Haushalte von Bad Birnbach richtet. Die Fragen fokussieren auf folgende Kategorien:

- Aktuelles Mobilitätsverhalten und Verkehrsmittelnutzung

- Aktive Nutzung des Pilotprojektes „Autonomer Kleinbus in Bad Birnbach“

- Haltung, Einschätzung und Bewertung des autonomen Kleinbusses in Bad Birnbach

- Haltungen, Erwartungen, Meinungen zu autonomem Fahren und autonom fahrenden Bussen generell

- Bedeutungszuweisung zu mobilitätsrelevanten gesellschaftlichen Themen, Werten und Normen (Umweltschutz, Datenschutz, technologische Innovationen, sozialer Gerechtigkeit) sowie zu alternativen Mobilitätskonzepten

- Nutzungsbereitschaft und Offenheit für autonome Kleinbussysteme in der Zukunft

Neben mehreren offenen Fragen wurden drei Fragenblöcke, welche die Bewertungs- und Einstellungsdimension betreffen, unter Verwendung einer 7er-Likert-Skala formuliert. Der Fragebogen wurde im Juli 2018 an alle Haushalte Bad Birnbachs verschickt. 420 Fragebögen wurden von den Anwohnern ausgefüllt, wovon 396 ausgewertet werden konnten (Rücklaufquote: ca. $40 \%$ ).

In der alltäglichen Verkehrsmittelnutzung der Befragten dominiert eindeutig der Pkw. Lediglich $5 \%$ der Befragten geben an, über kein eigenes Auto zu verfügen. Im Alltag wird der Pkw von $56 \%$ der Befragten täglich genutzt, während der ÖPNV (Bus, Nahverkehr) von keinem der Befragten als tägliches Verkehrsmittel genannt wird (Abb. 9.2). $78 \%$ der Befragten führen an, den ÖPNV nie als Fortbewegungsmittel zu wählen. Alternative Angebotsformen wie Sharingsysteme oder Rufbus werden sehr selten oder gar nicht im Alltag genutzt. Eine Klassifizierung nach Pkw-affinen Befragten, die den Pkw täglich oder mehrmals die Woche, aber nur selten oder nie den ÖPNV nutzen, sowie ÖV-affinen Befragten, die zumindest monatlich den öffentlichen Verkehr (ÖV) nutzen, soll in den folgenden Analysen für Vergleichszwecke verwendet werden. 


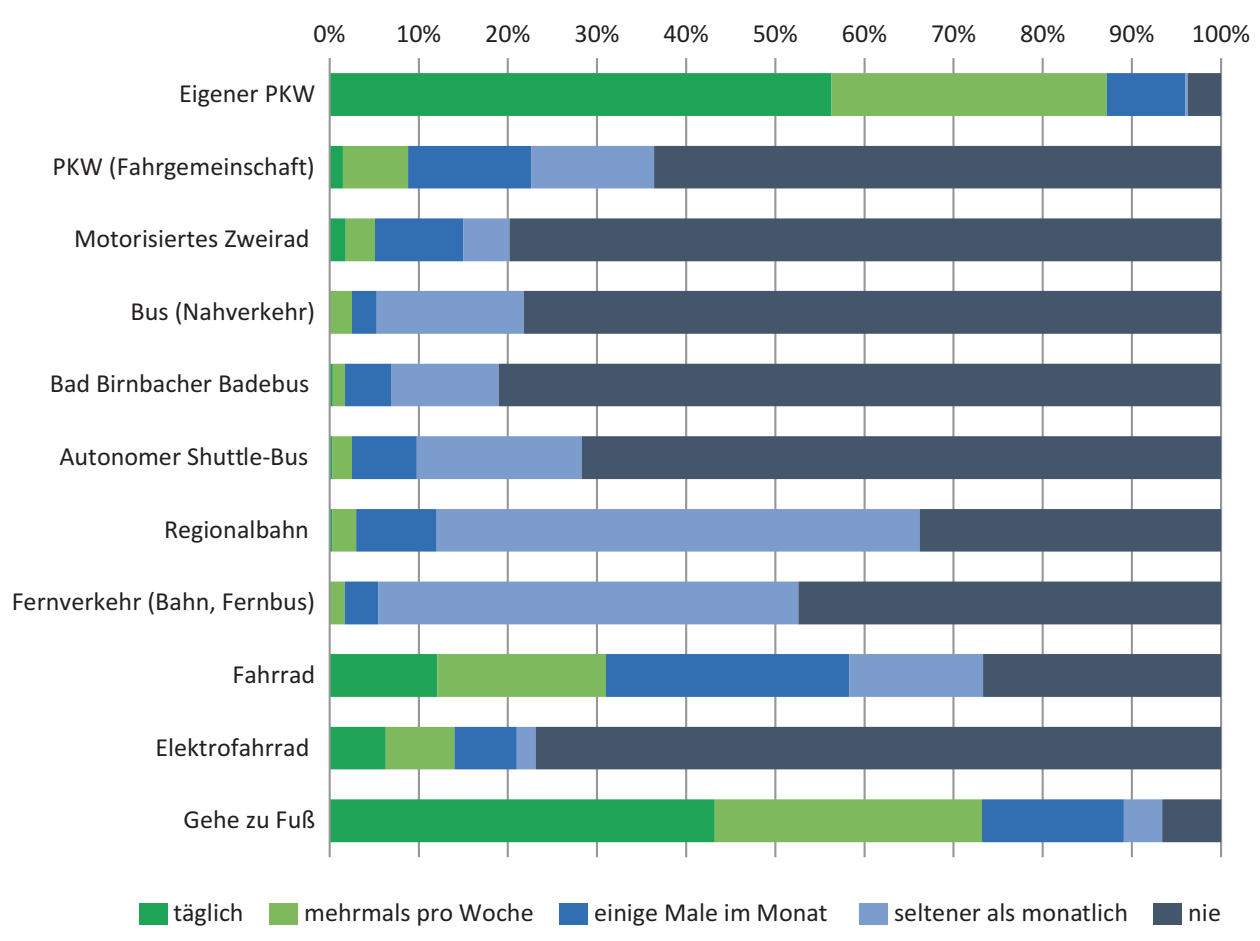

Abb. 9.2 Verkehrsmittelwahl im Alltag nach Intensität. (Quelle: eigene Darstellung)

\subsection{Einige empirische Ergebnisse zur Akzeptanz des automatisiert fahrenden Kleinbusses}

Die folgenden Auswertungen zu den verschiedenen Akzeptanzdimensionen orientieren sich am Phasenmodell Kollmanns (1998). Allerdings soll im Folgenden zunächst die Handlungsebene in Form der Handlungsakzeptanz des Pilotprojektes besprochen werden, um anschließend Hintergründe und Begründungen dafür auf der Einstellungsebene sowie mögliche Bewertungen für zukünftige Systeme auf der Nutzungsebene darzustellen.

\subsubsection{Handlungsdimension}

Sauer et al. (2005, S. 3) definieren in Anlehnung an die Überlegungen von Lucke (1995) Akzeptanz als ,,die positive Einstellung eines Akteurs einem Objekt gegenüber, wobei diese Einstellung mit Handlungskonsequenzen“ einhergeht. Wisser (2018, S. 50) sieht in dieser Definition als entscheidend an, dass ,Akzeptanz nicht nur anhand der positiven Bewertung, sondern auch zusätzlich anhand einer entsprechenden Handlung bestimmt wird. [...] Im Akzeptanzprozess resultiert die positive oder negative Einstellung gegenüber dem Objekt 
in einer annehmenden oder ablehnenden Handlung." Übertragen auf das Pilotprojekt kann unter der Handlungsdimension zum einen die Bewertung des Projektes als solches, aber zum anderen auch das aktive „Ausprobieren“ des automatisiert fahrenden Kleinbusses oder eben auch das Ausbleiben bzw. Ablehnen dieses aktiven Testens verstanden werden.

Das Pilotprojekt in Bad Birnbach wird von $65 \%$ der Befragten begrüßt, während $20 \%$ dem Projekt negativ gegenüberstehen, weitere $15 \%$ zeigen sich unschlüssig (Abb. 9.3). Personen, die regelmäßig im Alltag den ÖV nutzen (ÖV-Affine), stehen dem Projekt positiver (75\% Zustimmung) gegenüber, als die Vergleichsgruppe der fast ausschließlich Pkw-Fahrenden (Pkw-Affine) (64 \% Zustimmung).

Diese Bewertung des Gesamtprojektes zeigt sich auch auf der aktiven Handlungsebene in der bisherigen (Nicht-)Nutzung des automatisiert fahrenden Kleinbusses in Bad Birnbach (A.B.) (Abb. 9.4). Unter den Befürwortern des Projektes sind $52 \%$ schon einmal mit dem A.B. gefahren, während von den Ablehnern des Projektes nur $10 \%$ schon einmal mitgefahren sind (Anteil unter den Unschlüssigen: $33 \%$ ). Insgesamt hatten $58 \%$ der Befragten den automatisierten Kleinbus in Bad Birnbach zum Zeitpunkt der Befragung noch nicht, $27 \%$ einmal und $14 \%$ mehrfach genutzt. Diese aktive Handlung des Ausprobierens des A.B. durch die Befragten ist stark vom alltäglichen Verkehrsverhalten der Befragten

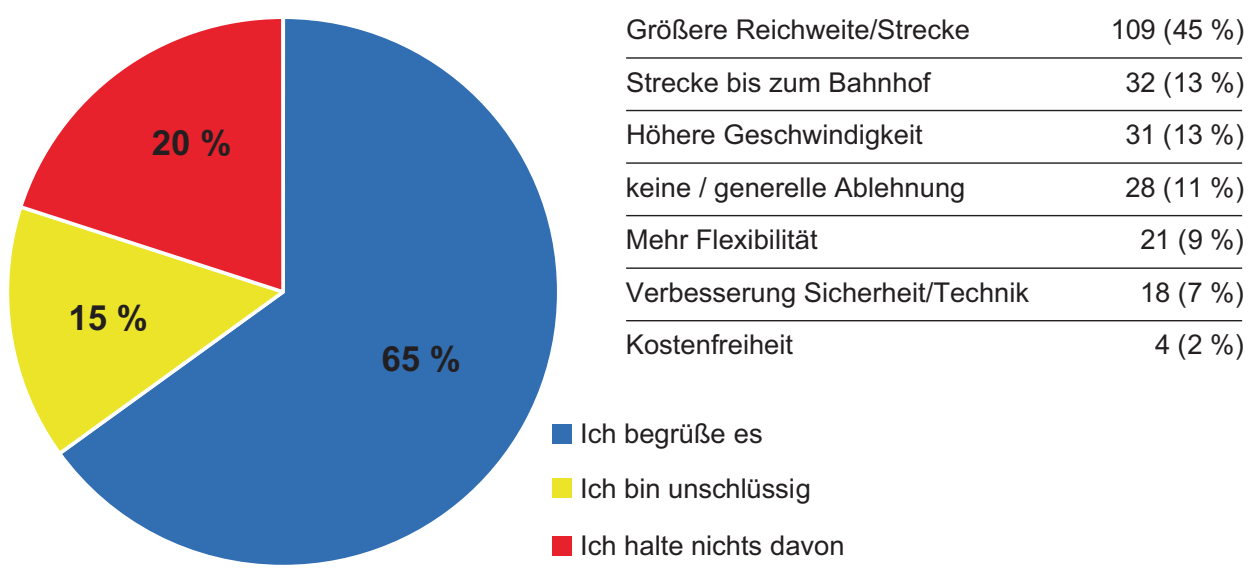

Abb. 9.3 Bewertung des Pilotprojektes und Verbesserungsvorschläge. (Quelle: eigene Darstellung)
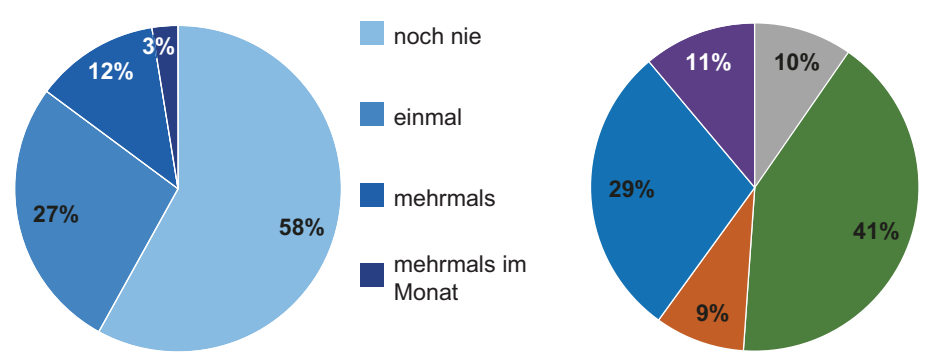

Neutraler Eindruck

Positiver Eindruck

Negativer Eindruck

Geringe Geschwindigkeit/

Geringe Reichweite

technische Probleme

Abb. 9.4 Häufigkeit der Nutzung des automatisiert fahrenden Kleinbusses und Eindrücke der ersten Fahrt. (Quelle: eigene Darstellung) 
abhängig. ÖV-Affine sind zu $61 \%$ schon mit dem A.B. gefahren, während unter den Pkw-Affinen dieser Anteil lediglich $35 \%$ ausmacht. Auch hinsichtlich der Intensität der im Alltag genutzten Verkehrsmittel existieren statistisch signifikante Unterschiede der A.B.-Nutzung (t-Test Mittelwertvergleich; Kontingenzkoeffizienten). Positiv auf die Nutzung des automatisierten Kleinbusses wirken die Merkmale Häufigkeit der Bahn- und Bus-Nutzung (jeweils $\alpha=0,01)$ und des zu Fuß-Gehens $(\alpha=0,05)$, während die alltägliche Pkw-Nutzung negativ mit der Nutzung des automatisierten Kleinbusses korreliert $(\alpha=0,01)$. Stark positiv korreliert auch die Nutzung des A.B. mit dem Alter der Befragten $(\alpha=0,01)$.

Die Eindrücke der „Tester“ von ihrer ersten Fahrt sind (in Antworten auf eine offene Frage) bei den meisten positiv (41\%), während $29 \%$ die geringe Reichweite und Geschwindigkeit kritisieren und $11 \%$ von technischen Schwierigkeiten berichten, wie dem unerwarteten Halten des Busses oder dem nicht barrierefreien Zustieg. $9 \%$ haben einen nicht weiter spezifizierten negativen Eindruck.

Gründe für die Mitfahrt sind vor allem Neugier (58\% der Nennungen) und technisches Interesse (26\%), 29 Befragte (13\% der Nennungen) finden die Strecke für ihre Ziele passend. Befragte, die den A.B. (noch) nicht ausprobierten, geben an, dass ihnen die Strecke zu kurz sei (38\% der Nennungen), der A.B. zu langsam ist (25\%) oder sie den öffentlichen Personennahverkehr ohnehin generell nicht nutzen würden (13\%). Unter Bedingungen, die erfüllt sein müssten, damit sie den A.B. nutzen würden, nennen die meisten, dass die Reichweite bzw. Strecke vergrößert werden sollte, der Anschluss des Bahnhofes erfolgen und die Geschwindigkeit erhöht werden müsste. Auch Aspekte wie mehr Flexibilität, Kostenfreiheit und die Verbesserung der Sicherheit und Technik werden genannt (Abb. 9.3).

\subsubsection{Einstellungsdimension}

Die Einstellungsdimension umfasst einem Akzeptanzobjekt gegenüber „Haltungen, Wertungen, Einschätzungen usw.“ (Fraedrich und Lenz 2015, S. 643). Eine positive Haltung, Einschätzung oder Bewertung ist im Verständnis von Handlungsabsichten und -bereitschaft die Voraussetzung für Handlungen (Schäfer und Keppler 2013, S. 11 f., Fraedrich und Lenz 2015, S. 644). Lucke (1995, S. 82) spricht im Kontext der Einstellungsdimension von ,latenter Akzeptanzbereitschaft“".

Für die analytischen Betrachtungen der Einstellungsebene kann man in Anlehnung an Fraedrich und Lenz (2015, S. 649 f.) ein Zwei-Ebenen-Kategorienschema verwenden, das diese für eine qualitative Inhaltsanalyse von Medien zur Akzeptanz von autonomem Fahren einsetzen. Übertragen auf die vorliegende Haushaltsbefragung ergibt sich folgende Kategorisierung von Haltungen, Wertungen und Einschätzungen der Befragten:

- Die objektbezogene, eher sachliche Ebene beinhaltet die wahrgenommenen Eigenschaften der Technologie, deren Entwicklungsmöglichkeiten und Potenziale.

- Auf einer subjektbezogenen, eher emotionalen Ebene werden Aussagen in direkten Bezug zur eigenen Person gesetzt und beinhalten Einstellungen, Bewertungen und Motivationen zum konkreten Akzeptanzobjekt (ebd.). 
Der Aspekt der Subjektbezogenheit kann sich jedoch auch stärker auf Bewertungen allgemeiner, weniger auf das konkrete Objekt bezogener Kriterien rund um Mobilität sowie zu gesellschaftlichen Normen beziehen.

- Deshalb soll die Kategorie „Mobilitätsrelevante Bewertungen“ zusätzlich verwendet werden. Sie beinhaltet allgemeinere Bedeutungszuweisungen der Befragten zu mobilitätsrelevanten gesellschaftlichen Themen, Werten und Normen (Umweltschutz, Datenschutz, technologische Innovationen, soziale Gerechtigkeit) sowie zu alternativen Mobilitätskonzepten.

Diesem Schema folgend wurden in der Haushaltsbefragung objekt-, subjekt- und mobilitätsbezogene Einstellungsfragen gestellt. Einige der Fragen sind nicht immer eindeutig zuordenbar und können sich inhaltlich in verschiedenen Kategorien wiederfinden, jedoch wurde, um Dopplungen zu vermeiden, in den graphischen Darstellungen eine fixe Zuordnung getroffen. In Abb. 9.5, 9.6 und 9.7 wird differenziert zwischen Pkw- und ÖV-affinen Befragten sowie zwischen Personen, die bereits mit dem automatisierten Kleinbus mitgefahren sind („Tester") oder nicht (,Nicht-Tester").

Hinsichtlich der objektbezogenen Eigenschaften gibt es größere Einstellungs- und Meinungsunterschiede zwischen „Testern“ und „Nicht-Tester“ als zwischen den Pkw- und ÖV-Affinen. Generell wird der A.B. vor allem als einfach in der Benutzung charakterisiert (Abb. 9.5). Dies artikulieren diejenigen, die den A.B. schon genutzt haben, statistisch signifikant häufiger als solche, die keine praktische Erfahrung mit dem A.B. haben. Weniger groß ist der Unterschied zwischen den ÖV- und Pkw-affinen Personen. Insgesamt schlechter bewertet werden die Vertrauenswürdigkeit und die Zuverlässigkeit. Auch bei diesen beiden Kriterien zeigen sich vor allem zwischen „Testern“ und „Nicht-Testern“ die größten Unterschiede. Starke Zustimmung erfährt die Aussage, dass der A.B. gerade für weniger mobile und ältere Bevölkerungsgruppen eine Chance für Mobilität bietet. Auseinander gehen die Meinungen bei den Vergleichsgruppen auch hinsichtlich des Potenzials des A.B., die Mobilität im ländlichen Raum zu sichern. Eher skeptisch fallen die Wertungen bzgl. des Substitutionspotenzials der A.B. für konventionelle ÖPNV-Systeme und sicherheitserhöhende Effekte für den Verkehr durch autonome Fahrzeuge aus. Auch in Rufbussysteme, in die der A.B. eingebunden werden könnte, werden keine hohen Erwartungen gesetzt.

In den Antworten zu den offenen Fragen spiegeln sich teilweise sehr verschiedene Haltungen und Einschätzungen zum automatisiert fahrenden Kleinbus wider (Tab. 9.1). So werden gegen autonom fahrende Busse vor allem technologische (unausgereifte Technik, zu geringe Geschwindigkeit) sowie die Sicherheit betreffende Bedenken vorgebracht, aber auch soziale (befürchteter Arbeitsplatzabbau) und finanzielle Kriterien werden genannt. Umgekehrt finden sich positive Einschätzungen in den Bereichen Ökologie, Sicherheit, Wirtschaft (finanzielle wie auch innovationsbezogene) und Soziales (Mobilität für Senioren, etc.; Unterhaltung).

Die hohe individuelle subjektbezogene Zustimmung spiegelt sich in der Aussage wider, dass die Idee des A.B. gefällt (Abb. 9.6). Das Vertrauen in den A.B. ist relativ groß, jedoch wird die Nützlichkeit für die jeweiligen Befragten sehr unterschiedlich bewertet. Hierbei unterscheiden sich die Aussagen der Vergleichsgruppen hoch signifikant $(\alpha=0,01)$. Vor allem die „Nicht-Tester“ bewerten Vertrauen und Nützlichkeit deutlich negativer als die 


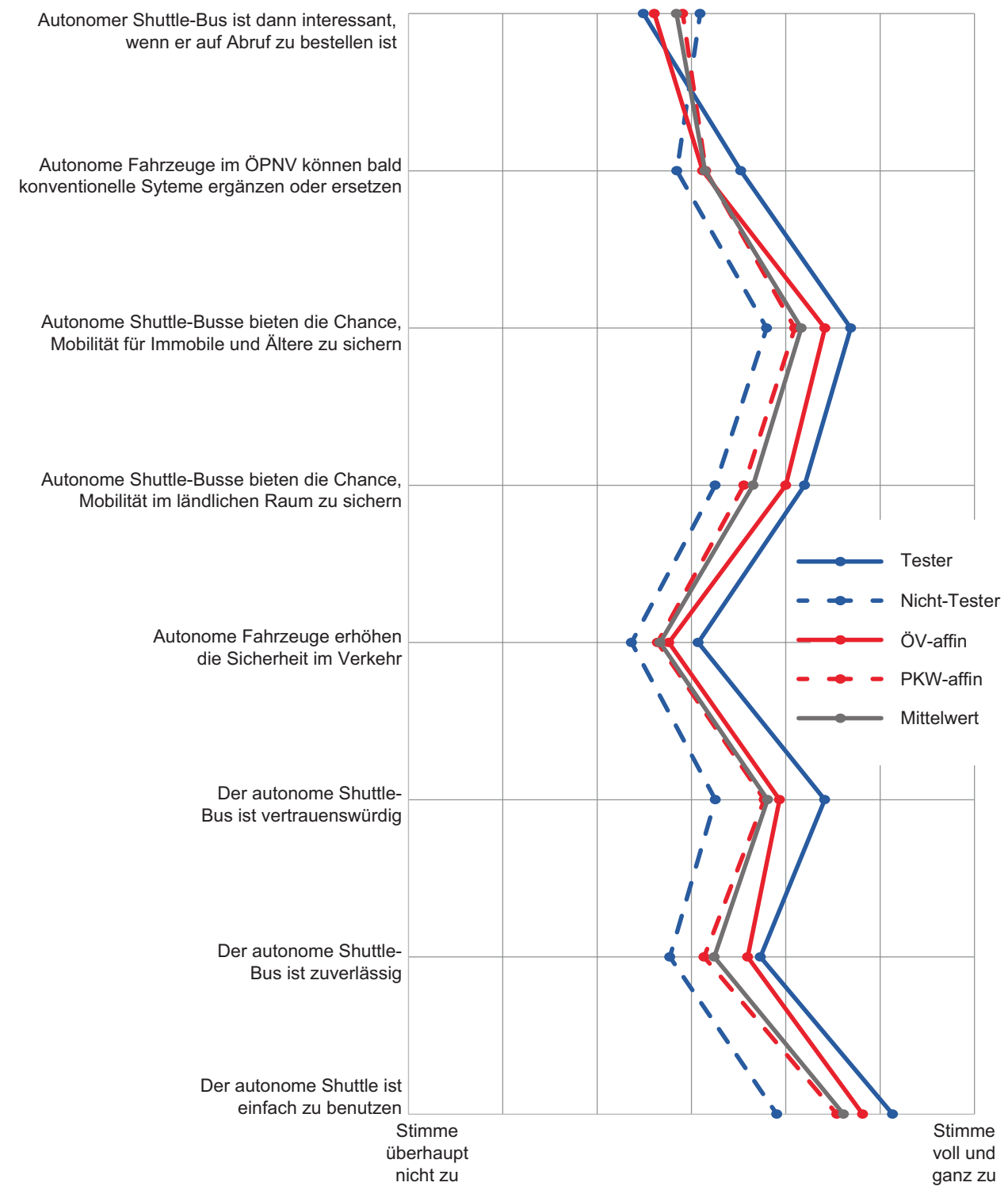

Abb. 9.5 Beurteilung objektbezogener Aussagen. (Quelle: eigene Darstellung)

anderen Gruppen. Die ÖV-Affinen und vor allem die „Tester“ schätzen vor allem das Potenzial der A.B. für sich selbst als sehr nützlich ein. Auch den Komfortgewinn, dass Fahrten für andere Tätigkeiten wie Arbeiten oder Lesen verwendet werden können, wird von den ÖV-Affinen höher geschätzt $(\alpha=0,01)$. Weniger als $32 \%$ der Befragten hegen Sicherheitsbedenken oder finden es gar unangenehm, einem A.B. zu begegnen (21\%). Für die meisten Befragten wird der A.B. aber erst dann interessant, wenn das Bedienungsgebiet erweitert wird. Zudem erwartet die Mehrheit keine Verbesserung der eigenen Produktivität. 
Für mich wird autonomer Shuttle-Bus erst interessant, wenn das Bedienungsgebiet erweitert wird

Den autonomen Bus zu benutzen, wäre nützlich für mich

Es ist mir egal, wenn eine Fahrt länger dauert, wenn ich dabei andere Tätigkeiten (lesen, arbeiten etc.) durchführen kann

Ich hätte Angst vor Hacker-Angriffen auf die Software, die autonome Fahrzeuge steuert

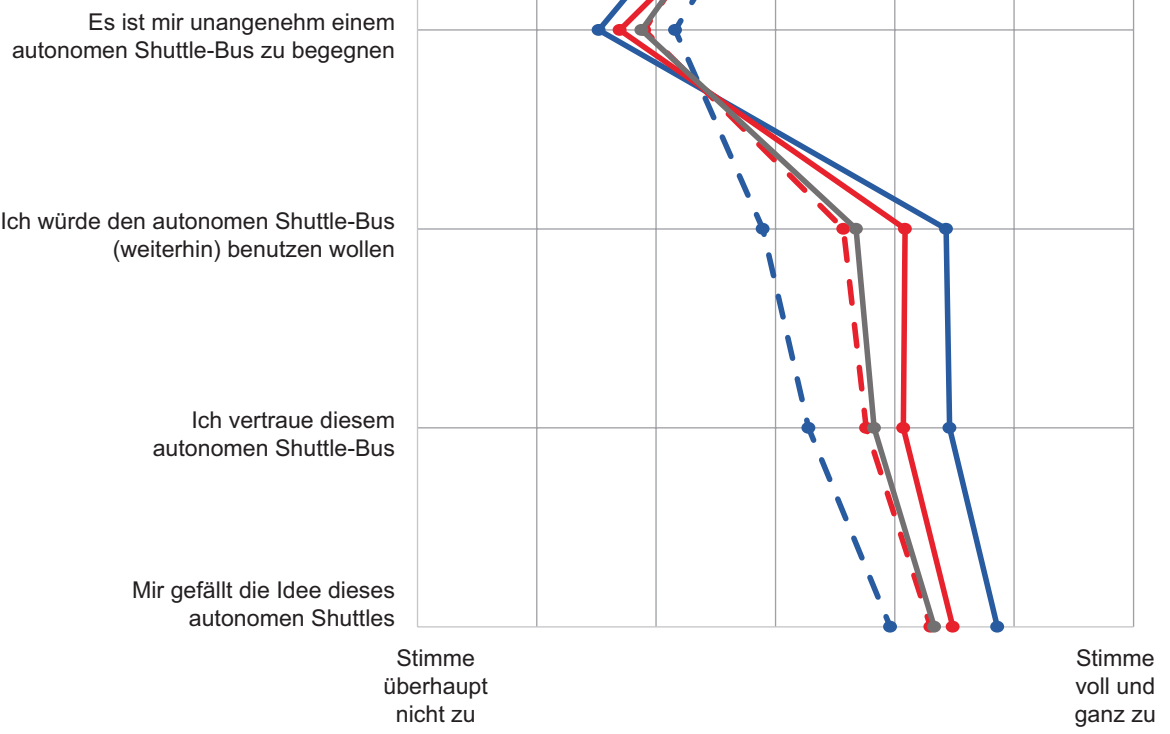

Abb. 9.6 Beurteilung subjektbezogener Aussagen zum A.B. (Quelle: eigene Darstellung)

Diese Bewertung des spezifischen Projektes in Bad Birnbach und zu autonomem Fahren generell kann um die Einstellung zu Themen der Mobilität im Allgemeinen erweitert werden (Abb. 9.7). In dieser vorwiegend auf Werthaltungen abzielenden Kategorie sind die Unterschiede zwischen den Vergleichsgruppen relativ gering. Die höchste Zustimmung erhielten die Aussagen „Umweltschutz ist mir wichtig“, „Ein eigener Pkw erhöht meine Flexibilität" sowie „Ich bin offen für neue Verkehrstechnologien“. Bei der Bewertung der Flexibilitätssteigerung durch einen eigenen Pkw ergeben sich statistisch signifikante Un- 


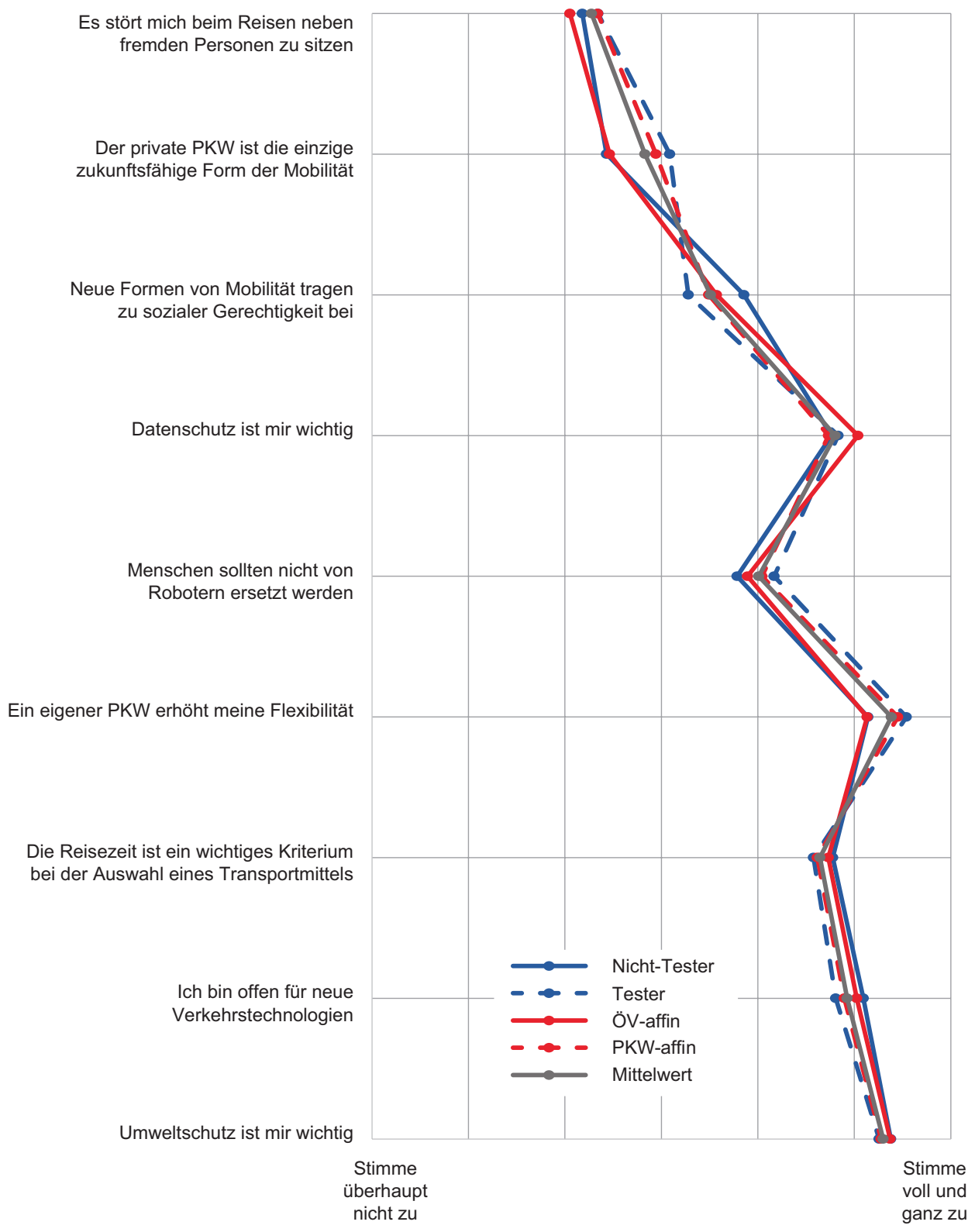

Abb. 9.7 Beurteilung subjektbezogener Aussagen zu Themen der Mobilität. (Quelle: eigene Darstellung)

terschiede zwischen „Testern“ und „ÖV-Affinen“ einerseits und „Nicht-Testern“ und „Pkw-Affinen“ andererseits (T-Test auf Mittelwertgleichheit: $\alpha=0,05$ ). Analoges gilt (allerdings mit insgesamt deutlich niedriger Zustimmungsrate) für die Bewertung einer Pkw-orientierten Zukunft der Mobilität (,Der private Pkw ist die einzig zukunftsfähige Form der Mobilität“). Am deutlichsten unterscheiden sich die Beurteilungen zwischen den 
Tab. 9.1 Artikulierte Gründe für und gegen automatisiert fahrende Kleinbusse. (Quelle: eigene Darstellung)

\begin{tabular}{l|l|l|l}
\hline Gründe für automatisiert fahrende Busse & \multicolumn{2}{l}{ Gründe gegen automatisiert fahrende Busse } \\
\hline Umweltschutz & $38(30 \%)$ & $\begin{array}{l}\text { Mangelnde Sicherheit/ } \\
\text { unausgereifte Technik }\end{array}$ & $63(33 \%)$ \\
\hline Erhöhung Flexibilität & $30(24 \%)$ & Angebot uninteressant & $39(20 \%)$ \\
\hline Kosteneinsparung & $24(19 \%)$ & Zu geringe Geschwindigkeit & $34(18 \%)$ \\
\hline $\begin{array}{l}\text { Mobilität für alle (Senioren, } \\
\text { Behinderte etc.) }\end{array}$ & $22(17 \%)$ & Arbeitsplatzabbau & $26(13 \%)$ \\
\hline Technische Weiterentwicklung & $9(7 \%)$ & Kosten & $19(10 \%)$ \\
\hline Unterhaltung & $3(2 \%)$ & Generelle Ablehnung & $12(6 \%)$ \\
\hline
\end{tabular}

beiden Gruppen „Tester“ und „Nicht-Tester“ hinsichtlich der Aussage „Neue Formen von Mobilität tragen zu sozialer Gerechtigkeit bei“.

\subsubsection{Nutzungsdimension}

Auf die Einstellungs- und Handlungsebenen folgt im Phasenmodell von Kollmann (1998, S. 113) die Nutzungsebene, bei der eine erfolgte Handlung (wie z. B. in anderen Kontexten der Produktkauf oder die Produktübernahme) in eine konkrete aufgabenbezogene bzw. problemorientierte Nutzung umgesetzt wird. „Die geplante Nutzungsintensität wird real umgesetzt oder den realen Gegebenheiten angepasst“" (Kollmann 2016, S. 411).

Übertragen auf den A.B. ist diese Ebene als konkrete Nutzung im Alltag und die Nutzung des A.B. in Mobilitätsroutinen zu interpretieren. Davon ist der A.B. allerdings noch ein gutes Stück entfernt. Insofern lässt sich diese Dimension nicht konkret messen, sondern bestenfalls können Nutzungsintentionen abgeschätzt werden. Solche Abschätzungen sollen im Folgenden aufgrund der Befragungsergebnisse vorgenommen werden. Dies geschieht, um einen groben Orientierungsrahmen für Nutzungspotenziale zu erhalten, gleichwohl sind mit einer derartigen Vorgehensweise einige Probleme verbunden.

Es sollen zunächst die objekt- und subjektbezogenen Einstellungsfragen aufgegriffen werden, die auf zukunftsgerichtete Aussagen abzielen. Eine zukünftige Nutzungsoption wurde mit der Frage untersucht, ob der A.B. benutzt werden würde, sollte er weiterhin angeboten werden. $22 \%$ artikulierten sich dazu eher negativ, $29 \%$ indifferent und $49 \%$ positiv. ${ }^{3}$ Erwartungsgemäß gibt es signifikante Unterschiede zwischen Befragten mit und ohne bisherige aktive Handlungserfahrung (Abb. 9.8). Diejenigen, die in Bad Birnbach schon mit dem A.B. gefahren sind, würden ihn großteils weiterbenutzen wollen (64\%), während dieser Anteil bei denjenigen, die noch keine Fahrt mit dem A.B. unternommen haben, bei lediglich $35 \%$ liegt. Allerdings wurde dazu eine Reihe von Bedingungen in offenen Antworten genannt. Insbesondere ein größeres Bediengebiet und in Bad Birnbach speziell die

\footnotetext{
${ }^{3}$ Die 7er-Likert-Skala wurde dazu in eine 3er-Skala transformiert (stimme überhaupt nicht zu, stimme nicht $\mathrm{zu}=$ Ablehnung; stimme eher nicht $\mathrm{zu}$, weder noch, stimme eher $\mathrm{zu}=$ indifferent; stimme zu, stimme voll und ganz zu = Zustimmung).
} 


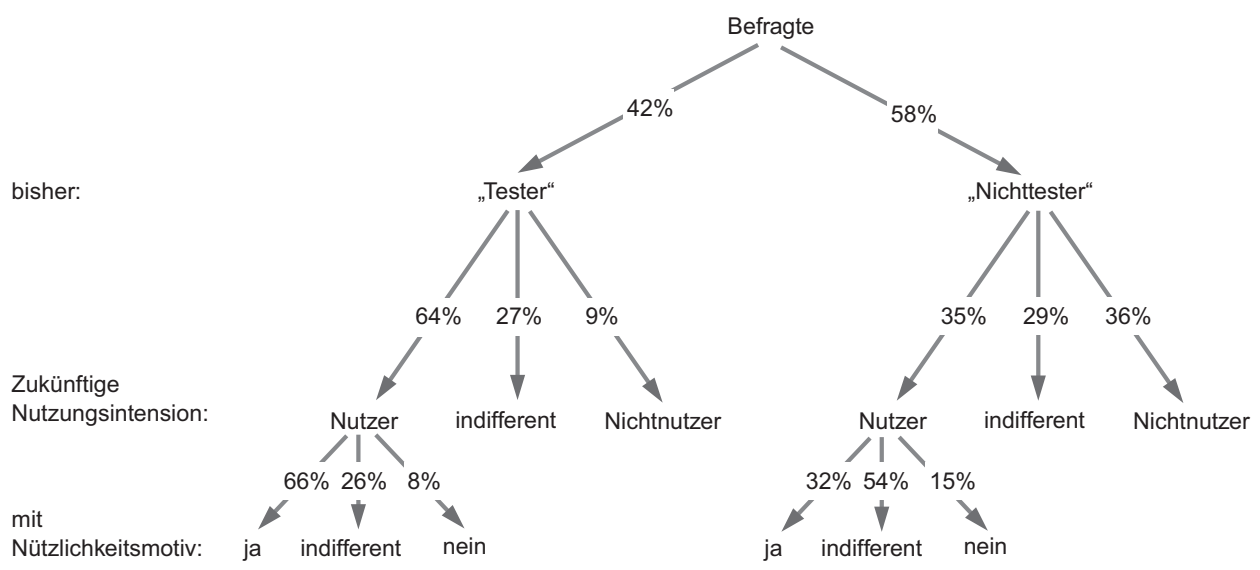

Abb. 9.8 Statistische Ableitung von Nutzungspotenzialen für den automatisiert fahrenden Kleinbus in Bad Birnbach aus der Befragung. (Quelle: eigene Darstellung)

Anbindung des Bahnhofs, aber auch eine höhere Fahrgeschwindigkeit, mehr Flexibilität und technische Reife wurden explizit mehrfach genannt. Auch die Einstufung des A.B. als für das Subjekt nützliches Verkehrsmittel ist relevant, da damit konkrete Mobilitätszwecke der Befragten, die über das bloße Testen hinausgehen, adressiert werden. Bezieht man diese Frage mit ein, so lassen sich grobe Nutzungspotenziale für den automatisiert fahrenden Kleinbus in Bad Birnbach ermitteln. Es zeigt sich, dass der Anteil der Befragten, die neben einer Nutzungsintention auch den A.B. zukünftig für sich als nützlich einschätzen, bei den „Testern“ signifikant höher ist als bei den „Nicht-Testern“. Aus Abb. 9.8 lässt sich ablesen, dass von 100 Befragten 42 den A.B. ausprobierten, von diesen zeigten 27 (64\%) eine weitere Nutzungsintention und aus dieser Gruppe wiederum verblieben 18 Personen (66 \%), die für sich im A.B. ein zukünftig nützliches Verkehrsmittel sehen. Aus der Gruppe der Nicht-Tester ließen sich entsprechend sechs Personen mit Nutzungsintention und positiver Nützlichkeitsbewertung selektieren. Diese deskriptiv aus den Befragungsdaten gewonnenen Informationen sollten nur als grobe Annäherungen an ein zukünftiges Nutzungspotenzial verstanden werden. Für exaktere Betrachtungen müsste das Produkt erst noch weitere technologische und organisatorische Weiterentwicklungen erfahren.

Um zukünftige potenzielle Nutzer des A.B. in Bad Birnbach nicht nur statistisch zu identifizieren, sondern auch in potenzielle Nutzergruppen zu typisieren, wurde weiterhin eine Clusteranalyse durchgeführt. Als Variablen finden auf die Zukunft gerichtete Aussagen der Befragten zu autonom fahrenden Bussen und zu Verkehrstechnologien wie auch zur Vertrauenswürdigkeit des A.B. Eingang in die Clusteranalyse (Ward-Methode) (Tab. 9.2).

Es wurden damit drei Gruppen identifiziert (Abb. 9.9). Eine Gruppe (Cluster 1) erreicht sehr hohe Zustimmungswerte bei den Items, die den autonomen Shuttle-Bus als mobilitätssichernde Zukunftstechnologie bezeichnen (,Autonome Shuttle-Busse bieten die Chance, Mobilität im ländlichen Raum zu sichern") und dabei große Offenheit für neue Verkehrstechnologien der Zukunft zeigen, die auch ohne privaten Pkw denkbar wären. Für die persönliche Nutzung eines autonom fahrenden Kleinbusses wäre entsprechender Bedarf vorhanden. 
Tab. 9.2 Variablen der Clusteranalyse (Skala 1: „stimme überhaupt nicht zu“, ... 7: „stimme voll und ganz zu“). (Quelle: eigene Darstellung)

\begin{tabular}{l|l|l}
\hline & Mittelwert auf 7er-Skala. & Std.-Abweichung \\
\hline $\begin{array}{l}\text { Den autonomen Bus zu benutzen, wäre nützlich } \\
\text { für mich }\end{array}$ & 4,0346 & 2,02244 \\
\hline $\begin{array}{l}\text { Ich würde den autonomen Shuttle-Bus } \\
\text { (weiterhin) benutzen wollen }\end{array}$ & 4,6759 & 2,09253 \\
\hline $\begin{array}{l}\text { Mobilität der Zukunft besteht aus autonomen } \\
\text { Fahrzeugen }\end{array}$ & 4,3497 & 1,90703 \\
\hline $\begin{array}{l}\text { Autonome Shuttle-Busse bieten die Chance, } \\
\text { Mobilität im ländlichen Raum zu sichern }\end{array}$ & 4,6541 & 1,90276 \\
\hline $\begin{array}{l}\text { Autonome Fahrzeuge im ÖPNV können in } \\
\text { wenigen Jahren konventionelle Systeme ergänzen } \\
\text { oder sogar ersetzen }\end{array}$ & 4,1445 & 1,89512 \\
\hline \begin{tabular}{l} 
Ich bin offen für neue Verkehrstechnologien \\
\hline $\begin{array}{l}\text { Der private Pkw ist die einzige zukunftsfähige } \\
\text { Form der Mobilität }\end{array}$
\end{tabular} & 5,9257 & 1,14064 \\
\hline \begin{tabular}{l} 
Der autonome Shuttle-Bus ist vertrauenswürdig \\
\hline
\end{tabular} & 4,8074 & 1,80537 \\
\hline
\end{tabular}

Die zweite Gruppe (Cluster 2) sieht dagegen autonome Fahrzeuge generell sowie auch autonom fahrende Busse nicht als Zukunftstechnologie für die Mobilität im ländlichen Raum an. Auch stufen sie die Benutzung des A.B., den sie als wenig vertrauenswürdig erachten, nicht als besonders nützlich ein. Der private Pkw wird von vielen als die einzige zukunftsfähige Form der Mobilität gesehen.

Die Befragten aus Cluster 3 sehen (sogar noch etwas intensiver als die Gruppe 1) in autonomen Fahrzeugen und Bussen eine Zukunftstechnologie, die Ergänzungs- und Substitutionspotenzial zu konventionellen Systemen im ÖPNV hat. Jedoch für sich selbst wird zumindest zum jetzigen Stand der Entwicklung keine sinnhafte Nutzung von A.B.s gesehen.

Für weitere Studien wären vertiefende Kategorisierungen der Items nützlich. So erbrachte eine Hauptkomponentenanalyse, ausgehend von 31 Variablen aus der Befragung zu den objekt- und subjektbezogenen Einstellungen und Bewertungen, sieben Hauptkomponenten, welche für die Bildung eines Kategorienschemas in zukünftigen Erhebungen hilfreich sein könnten (Abb. 9.10). Haushaltsbefragungen haben aber (wie jede empirische Erhebungsmethode) neben spezifischen Stärken auch Schwächen. So richtete sich der Fragebogen zwar an alle Haushalte und deren Mitglieder in Bad Birnbach, jedoch ist ein Bias zugunsten von Befragten, die dem automatisiert fahrenden Kleinbus positiv gegenüberstehen, nicht unwahrscheinlich.

\section{$9.4 \quad$ Fazit}

Eine Akzeptanzforschung zu einer sich erst in den Kinderschuhen befindlichen Technologie, wie den autonom fahrenden Bussen, kann hilfreich sein für die weitere technologische und organisatorische Weiterentwicklung des Systems sowie für entsprechende politische Entscheidungen. Es gibt jedoch auch einiges kritisch zu reflektieren. 


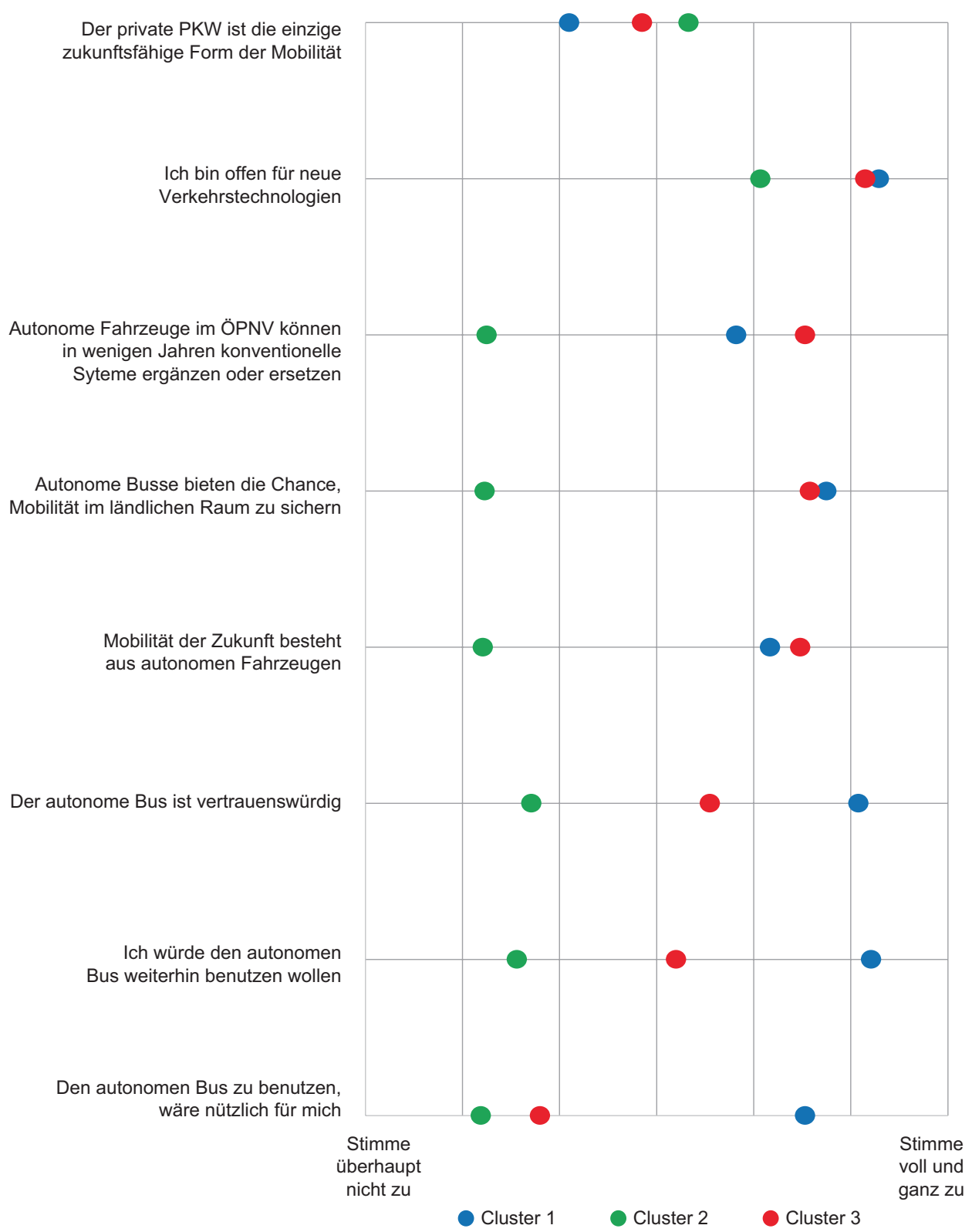

Abb. 9.9 Bildung von Clustertypen auf Basis der Einstellungsfragen. (Quelle: eigene Darstellung)

So wurden mehrfach von den Befragten Bedenken artikuliert, dass das Testgebiet in Bad Birnbach zu klein sei, um konkrete Aussagen über die Zukunftsfähigkeit von autonomen Bussen in ländlichen Räumen generell treffen zu können. Auch sei die Technologie im Vergleich zu konventionellen Systemen noch nicht wettbewerbsfähig (zu niedrige Geschwindigkeit, vereinzelte technische Probleme im Betrieb). Aspekte wie Sicherheit, Zu- 


\section{Offenheit/Begeisterung}

- Den autonomen Shuttle-Bus zu benutzen, ist ein erfreuliches Erlebnis

- Der autonome Shuttle ist einfach zu benutzen

- Der autonome Shuttle-Bus ist sinnvoll

- Der autonome Shuttle-Bus ist vertrauenswürdig

- Ich würde den autonomen Shuttle-Bus (weiterhin) benutzen wollen

- $\quad$ Autonome Shuttle-Busse bieten die Chance, Mobilität für immobile und ältere Menschen zu sichern

- $\quad$ Autonome Shuttle-Busse bieten die Chance, Mobilität im ländlichen Raum zu sichern

- Autonome Fahrzeuge im öffentlichen Personennahverkehr können in wenigen Jahren konventionelle Systeme ergänzen oder sogar ersetzen

\section{Neue (grüne) Technologien}

- Umweltschutz ist mir wichtig

- Ich bin offen für neue Verkehrstechnologien

\section{Konjunktiv der Nutzung}

- Den autonomen Bus zu benutzen, wäre nützlich für mich

- Für mich wird der Shuttle-Bus erst dann interessant, wenn das Bedienungsgebiet erweitert wird

- Die Benutzung des autonomen Shuttles würde meine Produktivität verbessern

\section{Sicherheitsbedenken}

- Ich habe Sicherheitsbedenken bei autonomen Shuttle-Bussen

- Ich hätte Angst vor Hacker-Angriffen auf die Software, die autonome Fahrzeuge steuert

\section{Nutzungsvorbehalte}

- Der private PKW ist die einzige zukunftsfähige Form der Mobilität

- Für mich wird der autonome Shuttle-Bus erst dann interessant, wenn ich inn auf Abruf zu mir bestellen kann

- Es stört mich beim Reisen unmittelbar neben fremden Personen zu sitzen

\section{Technikvorbehalte}

- Datenschutz ist mir wichtig

- Menschen sollten nicht von Robotern ersetzt werden

\section{Bedürfnis nach Flexibilität}

- $\quad$ Ein eigener PKW erhöht meine Flexibilität

- Die Reisezeit ist ein wichtiges Kriterium bei der Auswahl eines Transportmittels

Abb. 9.10 Hauptkomponenten aus Einstellungs- und Bewertungsfragen. (Quelle: eigene Darstellung) 
verlässigkeit, Erlebnischarakter und Komfort konnten zwar gut anhand des Pilotprojekts bewertet werden und auch in Zusammenhang mit eigenen subjektbezogenen Werten, Einstellungen und Haltungen zum Objekt selbst sowie zu allgemeineren Mobilitätsaspekten gebracht werden. Jedoch sind die von Grunwald (2005, S. 55 f.) formulierten Probleme und Kritikpunkte an der Akzeptanzforschung auch für das vorliegende Beispiel von Relevanz: „Es kann immer nur die jeweils gegenwärtige Akzeptanzsituation empirisch erfasst und in der betreffenden technikrelevanten Entscheidung berücksichtigt werden (auch das ist methodisch schon schwierig genug).“ Weil Technikakzeptanz auch zeitlich stark schwanken kann und damit instabil ist, ist Planungssicherheit weder für Investoren noch für Technikkonsumenten gegeben (Grunwald 2000, 2005, S. 56). Weitere empirische Akzeptanzanalysen anhand verschiedener Pilot- und Einsatzprojekte von automatisiert fahrenden Bussen sowie Panelstudien über längere Zeiträume anhand eines Projektes wären zukünftig erforderlich.

Auch dürften Probleme wie das von Grunwald (2005, S. 56) thematisierte Aggregationsproblem nur ansatzweise lösbar sein. Dieses besagt, dass individuelle Präferenzen hinsichtlich der Technikakzeptanz in einer pluralistischen, von Wertekonflikten durchzogenen Gesellschaft kaum zu einem konsistenten Gesamtbild zusammenzufügen sind. Um dieses Aggregationsproblem abzumildern, müssten möglichst vielfältige Meinungen und Einstellungen eingeholt werden. Dies ist mit der Berücksichtigung unterschiedlicher Akteurs- und Nutzergruppen und derer Perspektiven im gesamten Begleitforschungsprojekt durchaus angestrebt worden und spiegelt sich in der hier zugrunde liegenden Auffassung wider, dass die raum-zeitliche Ausbreitung einer verkehrstechnischen Innovation im Kontext einer gesellschaftlichen Einbettung geschieht, welche wiederum aus verschiedenen Dimensionen besteht (Kanger et al. 2018; Kap. 8, Appel et al.). Die Akzeptanz und Nutzung des autonom fahrenden Busses durch die Bewohner unter Berücksichtigung von deren Kontexten ist hier zwar nur ansatzweise erfolgt. Gerade die Gegenüberstellung der Aussagen von Nutzern und Nicht-Nutzern und deren sehr unterschiedlichen Meinungen und Bewertungen von mobilitätsbezogenen Themen ergeben jedoch ein sehr differenziertes Bild zur Bedeutung von personenbezogenen Kontexten.

Die hier im Speziellen auf die Haushalte fokussierte Herangehensweise einer schriftlichen Haushaltsbefragung ist ein sinnvoller Weg. Sie erbrachte auch zahlreiche Einsichten in die unterschiedlich sich nach Gruppen differenzierenden Dimensionen von Akzeptanz. Methodisch haben sich die vielfältigen Einstellungsfragen als aussagekräftig erwiesen.

\section{Literatur}

Fraedrich E, Lenz B (2015) Gesellschaftliche und individuelle Akzeptanz des autonomen Fahrens. In: Maurer M, Gerdes JC, Lenz B, Winner H (Hrsg) Autonomes Fahren. Technische, rechtliche und gesellschaftliche Aspekte. Berlin/Heidelberg, S 639-660

Grunwald A (2000) Technology Policy Between Long-Term Planning Requirements and ShortRanged Acceptance Problems. In: Grin J, Grunwald A (Hrsg) Vision assessment: shaping technology in 21 st century society. Heidelberg, S 99-148 
Grunwald A (2005) Zur Rolle von Akzeptanz und Akzeptabilität von Technik bei der Bewältigung von Technikkonflikten. In: Technikfolgeabschätzung - Theorie und Praxis, 14/3, S 54-60

Hüsing B, Bierhals R, Bührlen B, Friedewald M, Kimpeler S, Menrad K, Wengel J, Zimmer R, Zoche P (2002) Technikakzeptanz und Nachfragemuster als Standortvorteil. Karlsruhe

Kanger L, Geels FW, Sovacool B, Schot J (2018) Technological diffusion as a process of societal embedding: Lessons from historical automobile transitions for future electric mobility. Transportation Research Part D: Transport and Environment. doi: https://doi.org/10.1016/j.trd.2018.11.012

Kollmann T (1998) Akzeptanz innovativer Nutzungsgüter und -systeme. Wiesbaden

Kollmann T (2016) E-Entrepreneurship. Grundlage der Unternehmensgründung in der Digitalen Wirtschaft. Wiesbaden, 6. Aufl

Lucke D (1995) Akzeptanz: Legitimität in der „Abstimmungsgesellschaft“. Opladen

Sauer A, Luz F, Suda M, Weiland U (2005) Steigerung der Akzeptanz von FFH-Gebieten (=BfN-Skripten 144). URL (11.02.2019): http://www.bfn.de/fileadmin/MDB/documents/ skript144.pdf

Schäfer M, Keppler D (2013) Modelle der technikorientierten Akzeptanzforschung. Überblick und Reflexion am Beispiel eines Forschungsprojekts zur Implementierung innovativer technischer Energieeffizienz-Maßnahmen. Zentrum Technik und Gesellschaft: discussion paper Nr. 34/2013

Schweizer-Ries P, Rau I (2010) Aktivität und Teilhabe - Akzeptanz Erneuerbarer Energien durch Beteiligung steigern. Projektabschlussbericht

Wisser K (2018) Gebäudeautomation in Wohngebäuden (Smart Home). Wiesbaden

Open Access Dieses Kapitel wird unter der Creative Commons Namensnennung 4.0 International Lizenz (http://creativecommons.org/licenses/by/4.0/deed.de) veröffentlicht, welche die Nutzung, Vervielfältigung, Bearbeitung, Verbreitung und Wiedergabe in jeglichem Medium und Format erlaubt, sofern Sie den/die ursprünglichen Autor(en) und die Quelle ordnungsgemäß nennen, einen Link zur Creative Commons Lizenz beifügen und angeben, ob Änderungen vorgenommen wurden.

Die in diesem Kapitel enthaltenen Bilder und sonstiges Drittmaterial unterliegen ebenfalls der genannten Creative Commons Lizenz, sofern sich aus der Abbildungslegende nichts anderes ergibt. Sofern das betreffende Material nicht unter der genannten Creative Commons Lizenz steht und die betreffende Handlung nicht nach gesetzlichen Vorschriften erlaubt ist, ist für die oben aufgeführten Weiterverwendungen des Materials die Einwilligung des jeweiligen Rechteinhabers einzuholen.

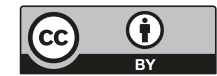

